\title{
Przewalski's Horses (Equus ferus przewalskii) Responses to Unmanned Aerial Vehicles Flights under Semireserve Conditions: Conservation Implication
}

\author{
Vanessa Lu $\mathbb{D}^{1},{ }^{1}$ Feng Xu $\mathbb{D}^{2},{ }^{2}$ and Mardan Aghabey Turghan $\mathbb{D}^{2}$ \\ ${ }^{1}$ The Thacher School, 5025 Thacher Road, Ojai, CA 93023, USA \\ ${ }^{2}$ State Key Laboratory of Desert and Oasis Ecology, Xinjiang Institute of Ecology and Geography, Chinese Academy of Sciences, \\ Urumqi 830011, China \\ Correspondence should be addressed to Mardan Aghabey Turghan; mardan@ms.xjb.ac.cn
}

Received 19 December 2020; Revised 23 May 2021; Accepted 11 August 2021; Published 28 August 2021

Academic Editor: Lesley Rogers

Copyright (c) 2021 Vanessa Lu et al. This is an open access article distributed under the Creative Commons Attribution License, which permits unrestricted use, distribution, and reproduction in any medium, provided the original work is properly cited.

Recent technological innovations have led to an upsurge in the availability of unmanned aerial vehicles (also known as drones and hereafter referred to as UAVs) - aircraft remotely operated from the ground-which are increasingly popular tools for ecological research, and the question of this study concerns the extent to which wildlife responses might allow aerial wildlife monitoring (AWM) by UAVs. Our experiment tests the hypothesis that the wildlife-UAVs interaction depends strongly on flight altitude that there may be a lowest altitude range for which the ungulates are not exceedingly disturbed, dictating a practically achievable level of discernibility in flight observation, for this question might influence the future viability of the UAVs in the study and protection of the other wildlife in China's semiarid ecosystem. We examined the behavioral responses of a group of enclosed Przewalski's horses (Equus ferus przewalskii) to the presence of different in-flight UAVs models by conducting flights at altitudes ranging from 1 to 52 meters and recorded the heights at which each horse reacted to (noticed and fled) the UAVs. All horses exhibited a stress response to UAVs flights as evidenced by running away. The results suggest strong correlations between flight altitude and response across the different subjects that adults generally noticed the UAVs at the larger heights $(20.58 \pm 10.46 \mathrm{~m})$ than the immature $(4.67 \pm 0.87 \mathrm{~m})$. Meanwhile, reaction heights of females $(15.85 \pm 6.01 \mathrm{~m})$ are smaller than that of males $(26.85 \pm 18.52 \mathrm{~m})$. Supported by their biological roles in herds (e.g., males must give protection to his entire herd while females are purely responsible for their offspring), our results also show that age, closely followed by gender, are the two most significant elements that determine a horse's level of alertness to the UAVs. This research will help future scientists to better gauge the appropriate height to use a drone for animal observation in order to minimize disturbance and best preserve their natural behavior.

\section{Introduction}

1.1. Przewalski's Horses. Przewalski's horses (Equus ferus przewalskii), also known as Mongolian wild horses or Dzungarian Horses, are the last surviving subspecies of wild horses. Although initially sighted in the 15th century, these horses were not scientifically documented until 1880, and these horses were sighted in the Mongolian Gobi Desert, by a Russian officer, Colonel Nikolai Przhevalsky, so the horses were named after him $[1,2]$. After being captured and transferred to Europe in the early 1900s, Przewalski's horses soon became endangered due to habitat loss, over hunting, and competition with livestock [1-3]. The last recorded sighting of a wild Przewalski's horse occurred in the Dzungarian Gobi of Mongolia in 1969 [4], and since then, this species has been extinct in the wild with only a few remnant populations existing in small captive breeding herds in Western countries, making the survival of the taxon possible [5-7]. All Przewalski's horses alive today are descendants from only 13 individuals that were the nucleus for captive breeding $[1,3,6,8,9]$. After another 20 years of captive breeding on four different continents, including Asia, the total number of horses rose to almost 1000 individuals $[3,6,7,10-14]$, and many organizations have since then also attempted to release the horses in semiwild habitats such as the 
Kalamaili Nature Reserve in Xinjiang, China. Although this may be a step in the right direction, these populations remain somewhat dependent on human support for their survival. Therefore, recommendation for the success of this project states to limit the number of domestic animals in the area, in order to ensure a long-term self-sustaining population of Przewalski's horses [1-3].

1.2. Previous Use of Unmanned Aerial Vehicles in Studies of Wildlife Research. Unmanned aerial vehicles (UAVs) have the potential to revolutionize the way in which research is conducted in many scientific fields $[15,16]$. UAVs have proven to be useful devices for the observation of wildlife, in particular the production of systematic data with high spatial and temporal resolution because the devices can access remote or difficult terrain [17], collect large amounts of data for lower cost than traditional aerial methods, and facilitate observations of species that are wary of human presence [18]. Currently, despite large regulatory hurdles, UAVs are being deployed by researchers and conservationists to monitor threats to biodiversity, collect frequent aerial imagery, estimate population abundance, and deter poaching [19-24], but with the widespread increase in UAV flights, it is critical to understand whether UAVs act as stressors to wildlife and to quantify that impact. It is likely that UAVs could also have unwanted and unanticipated risks on wildlife and their delicate ecosystems. Research has shown that retaliation against UAVs by terrestrial mammals was different from that of marine mammals and aquatic birds $[25,26]$. For example, a study into the free-roaming American black bears (Ursus americanus) proved that the presence of UAV flights brings significant distress to the physiological state, which often does not manifests itself in terms of behavioral changes, proving difficult for observers to discern [27]. Furthermore, a study on guanacos (Lama guanicoe) revealed that low-flying UAVs at any speed, as well as high-flying UAVs at an accelerated velocity, caused a disturbance to the guanacos' behavior [28].

Although UAVs have potential for success, a great deal of uncertainty still surrounds their use, and scientists must be cautious of the impact that they bring to each new investigation, especially when endangered species or ecologically sensitive habitats are involved [27]. Moreover, it is vital that UAVs are manufactured and selected to minimize visual and audio stimuli in order to reduce disturbance of wildlife. Shape, volume, and color are all factors to be taken into consideration thus to mimic nonthreatening wildlife native to the studied habitat in order to decrease disruption [29]. Sporadic movements and threatening or alarming trajectories should be avoided at all costs, and if an operation proves to be excessively disruptive, it must be ceased immediately [27]. With these basic regulations in place to ensure safety and ethicality, UAV flight are sure to make steady progress and evolve into more effective devices for the study of animal behavior.

1.3. Summary and Hypothesis. As the last surviving subspecies of wild horses, Przewalski's horses are a great subject of interest to the scientific community. Despite extensive studies especially on their social behavior and time budget [30-38], Przewalski's horses remain somewhat of a mystery to scientific world today, primarily because of their rarity. The purpose of our study was to better understand Przewalski's horses, in particular their response to UAVs under semireserve conditions. Previous studies of Przewalski's horses and domestic and feral horses (Equus caballus) have shown left side bias in vigilance responses with horses reacting sooner when approached from the left side by novel stimuli [39-41]. In this study, we wanted to look at the influence of the height of novel stimuli. We specifically focused on discerning how individual horses reacted to the height of UAVs and which factors influenced their reactions, whether it be age, gender, or the herd that they resided in. Our hypothesis is that wildlife-UAVs interaction depends strongly on flight altitude, that flying too low could excessively disturb them and that there may be a lowest altitude range for which the ungulates are not exceedingly disturbed.

Additionally, we hoped to learn the height at which UAV flights could operate without disturbing the horses and whether UAVs can be a reliable tool in the observation of their behavior and other wild wildlife. We also hypothesized that horses would respond to the UAV flight in one of four ways: no discernable responses (positive) and discernable responses (negative) (i.e., attention, eye/head movement and/or turning of the upper body, increased movement rates, and/or moving away slowly from the UAVs). We also hypothesized that the degree of responses would vary with respect to age and gender.

\section{Materials and Methods}

The design of the experiment began determining a range of flight altitudes. The range of flight altitudes was determined through preliminary tests, flying at altitudes from 1 meter to 100 meters in $10 \mathrm{~m}$ increments. We determined that $1-5 \mathrm{~m}$ was a suitable lower bound because of safety concerns and that 50-60 m was a suitable upper bound because negative response had already significantly dropped off by that altitude.

2.1. Study Area. This study was conducted at three separate enclosures of the Wild Horse Breeding Research Center of Xinjiang Uygur Autonomous Region, China $\left(44^{\circ} 12^{\prime} 20.5^{\prime} \mathrm{N}\right.$, $\left.88^{\circ} 44^{\prime} 52.8^{\prime} \mathrm{E}\right)$. This area has a semiarid, desert climate with great seasonal temperature differences: the hottest recorded summer temperatures $40^{\circ} \mathrm{C}, 104^{\circ} \mathrm{F}$, and winter temperatures often fall below $-15^{\circ} \mathrm{C}$. To maintain natural breeding patterns and social structures of Przewalski's horses, the horses typically remained in their native herd following birth to be raised by their mothers. Once they reached a mature age, they would be reallocated to herds corresponding to their genders. Occasionally, mares would be regrouped to create a novel mixed herd, while stallions would be reintroduced to such mixed herds to determine dominance. The defeated stallion returns to his single-sex herd, while the victorious stallion gains control over his new mixed-sex herd.

2.2. Data Collection. The unmanned aerial vehicle (UAVs) we used was a Mavic 2 Zoom drone, powered by a $1 / 2.3$ inch $12 \mathrm{MP}$ sensor with up to $4 \mathrm{x}$ zoom, including a $2 \mathrm{x}$ optical 
zoom $(24-48 \mathrm{~mm})$ to capture all shorts from wide angle to midrange. The $4 \mathrm{x}$ lossless zoom and $2 \mathrm{x}$ optical zoom enabled a closer, high-definition view of faraway subjects while maintaining our distance to decrease the disruption to our horses. The Mavic 2 cameras utilized DJI's latest 3-axis gimbal technology and recorded videos at higher bitrates with advanced H.265 compression. Videos in H.265/HEVC codec maintain $50 \%$ more information than videos in H.264/ AVC, which leads to better preserved details allowing us to clearly recognize behavioral signals in our horses. Additionally, FOC sinusoidal drive ESCs and low-noise propellers reduce flight noise, thus reducing disturbance to the horses. Binoculars (10x magnification) were used when needed. Horses were followed on foot and watched at distances of 30-100 m. Individual horses were identified using sex, size, color, and distinguishing markings.

UAV flights were conducted from August 1, 2020, to December 08, 2020, at consistent times during daylight hours in order to reduce the influence of other factors that may affect the reaction times of Przewalski's horses. The research team consists of four personnels: (1) a pilot, (2) a primary observer, (3) a ground camera operator, and (4) a data recorder. The pilot was responsible for flying the UAVs. Only one horse was scored when the drone was flown over a herd. Flights were made of 62 horses including 53 adults and nine immature horses (adult male, $N=9$; immature male, $N=5$; adult female, $N=44$; and immature male, $N=4$ ) living under natural social conditions at three separate enclosures. The ages of the horses were obtained from records of the reserve association (horses ranged from 1 day to 2 years were categorized as immature until they had left their natal band). Since these horses have been studied extensively, they were habituated to observation and were not highly vigilant and reactive to the presence of the observer, who could watch from a distance up to as little as $20-50 \mathrm{~m}$ while remaining stationary.

The UAVs were launched approximately $100 \mathrm{~m}$ from the targeted location of the horse. The total dataset consisted of 62 flights that ranged in altitude from 1 to 100 meters. Each flight began with taking off from a launch point, flying about $100 \mathrm{~m}$ high from a focal individual animal, changing altitude as appropriate when passing over the subject. The UAVs were flown over the animal or herd at $10 \mathrm{~m} / \mathrm{s}$. The next pass was flown at another randomly selected altitude. If an affirmative response was invoked, the drone would wait at a distance, while the animals would settle back to a sedentary behavior.

We used the live video feed which also offers a recorded video after a completed flight and the location stamp, which informs us of the exact height and angle of the drone to determine the vertical distance from our subjects, as well as the exact measurements at which they individually displayed the two levels of reaction that we were measuring. To avoid confusion, the term "response" is used here for all levels of behavioral responses, as it is for a broad range of species to refer to an animal interrupting feeding, by lifting or cocking its head, and to attend to its surroundings [41]. Level 1, known as the alert height, was determined through the eye and ear movements of Przewalski's horses; these reactions can range from subtle ear swivels indicating auditory influence to indirect glances at the UAVs. Once a subject displays any of these reactions, it becomes aware of the existence of the drone, and the height of the UAVs was recorded as the alert height. Level 2, known as the run away height, was the height of the UAVs at which the subject physically displaced itself to avoid the UAVs.

2.3. Data Analysis. Data were tested for normality with the one sample Kolmogorov-Smirnov test. Because all of the data showed a normal distribution, we used the $t$-test to detect the differences of alert height and flight initiation height between the age and sex. We then used the general linear model to test the effect of age, sex, and their interaction on alert height and flight initiation height. We accepted statistical significance at the level of $p>0.05$, and all the data were analyzed using the SPSS 19.0 statistical package.

\section{Results}

Przewalski's horses responded to UAV flights in all 62 flights. Tables 1 and 2 display the comparative makeup of each response for the different altitudes. First, notice that the positive responses (no discernable movement and alert) increases with altitude, while the negative responses (discernable movement and alert) decrease with altitude. The results help us illustrate the general pattern of less negative response at higher altitudes supporting the hypothesis that altitude plays a large role in animal response from UAVs. Next, notice that the degree of response varied with respect to age and gender: the alert height (height for horse to notice UAVs) and run away height (height for horse to move away) of adult horses ranged 11-52 and 1-36 meters while that of immature ranged 3-6 and 1-2 meters, respectively. The result also showed that immature compared to adults showed a greater effect in males than in females (Table 1). Additionally, alert height and run away height for female of all tested herds ranged 8-30 and 1-4 meters, while for male ranged $29-52$ and 4-36 meters, respectively, showing that male horses are more alert than the females (Table 2). In summary, age, sex, and their interaction have significant effects on alert height and run away height (Table 3).

\section{Discussion}

With the widespread increase in UAV flights, it is critical to understand whether UAVs act as stressors to wildlife and to quantify that impact [27]. Prior to this study, [27] assessed effects of UAV flights on movements and heart rate responses of free-roaming American black bears and concluded that UAV flights induced strong behavioral as well as physiological responses, but most bears did not respond behaviorally by increasing movement rates or moving to a different location. However, horses responded rather strongly to UAV flights in all 62 tests in this study. Przewalski's horses were unhabituated to the UAVs, and the study was conducted in the presence of stress-inducing events that occur naturally in the environment. The results 
TABLE 1: Comparison of alert height and run away height of Przewalski's horses.

\begin{tabular}{|c|c|c|c|c|c|c|}
\hline & Variables & Number & Alert height $(\mathrm{m}) \pm \mathrm{SE}$ & $t$-test & Run away height $(\mathrm{m}) \pm \mathrm{SE}$ & $t$-test \\
\hline \multirow{2}{*}{ Males } & Immature males & 5 & $5.0 \pm 0.71$ & $P<0.001$ & $1.40 \pm 0.55$ & $P=0.003$ \\
\hline & Adults males & 9 & $40.33 \pm 9.38$ & & $18.67 \pm 12.80$ & \\
\hline \multirow[t]{2}{*}{ Females } & Immature females & 4 & $4.50 \pm 0.50$ & $P<0.001$ & $1.25 \pm 0.50$ & $P=0.130$ \\
\hline & Adults females & 44 & $16.68 \pm 5.26$ & & $2.16 \pm 0.83$ & \\
\hline
\end{tabular}

Alert height, height for horse to notice UAVs; run away height, height for horse to move away (means with standard errors). "Number" refers to the number of flights: only one horse was scored when the drone was flown over a herd.

TABLE 2: Comparison of alert height and run away height between age and sex of Przewalski's horses.

\begin{tabular}{|c|c|c|c|c|c|c|}
\hline & Variables & Number & Alert height $(\mathrm{m}) \pm \mathrm{SE}$ & $t$-test & Run away height $(\mathrm{m}) \pm \mathrm{SE}$ & $t$-test \\
\hline \multirow{2}{*}{ Age } & Immature & 9 & $4.67 \pm 0.87$ & $P<0.001$ & $1.22 \pm 0.4$ & $P=0.013$ \\
\hline & Adults & 53 & $20.58 \pm 10.46$ & & $5.18 \pm 8.82$ & \\
\hline \multirow{2}{*}{ Sex } & Males & 14 & $26.85 \pm 18.52$ & $P=0.025$ & $11.50 \pm 12.61$ & $P=0.01$ \\
\hline & Females & 48 & $15.85 \pm 6.01$ & & $2.11 \pm 0.84$ & \\
\hline
\end{tabular}

Alert height, height for horse to notice UAVs; run away height, height for horse to move away (means with standard errors). "Number" refers to the number of flights: only one horse was scored when the drone was flown over a herd.

TABle 3: Effects of age, sex, and their interaction on alert height and run away height.

\begin{tabular}{lcc}
\hline Factors & Alert height $(\mathrm{m})$ & Run away height $(\mathrm{m})$ \\
\hline \multirow{2}{*}{ Age } & $F=19.345$ & $F=15.111$ \\
& $P \leq 0.001$ & $P=0.002$ \\
Sex & $F=13.383$ & $F=28.510$ \\
& $P=0.001$ & $P=0.01$ \\
Interaction of age and sex & $F=13.133$ & $F=17.324$ \\
\end{tabular}

Alert height, height for horse to notice UAVs; run away height, height for horse to move away (means with standard errors).

suggest strong correlations between flight altitude and response which support our hypothesis 2 that UAVs induced behavioral responses, including relocation (moving out of the way of the UAV but still in sight) and flee (movement from sight) as well as eye/head movement and/or turning of the upper body.

Hypothesis 3 was also strongly supported by our result. The strength of the responses varied among sex and age, and our results suggest that age and gender are the significant determining factors of a horse's reaction to UAVs. Mature horses were significantly more vigilant of the UAVs than immature as demonstrated by the higher heights at which adult horses were alerted to the flying UAVs compared to the immature. Therefore, this can be attributed to the lack of experience of younger horses; thus, a lower level of response, which supports previous data on the behavior of Przewalski's horses, will be further discussed later. The results also sets gender (exclusive to mature horses) as the second most influential factor of UAVs reaction height for the fact that alert height for female of all tested herds was lower than that of any male horse.

Both of these conclusions are not only supported by our data but also justified by the works of other scientists, as Kolter mention the role of a stallion as the protector of his herd, especially one that includes mares and foals [26]. An integral element of his responsibility depends on his ability to fend off unknown creatures, which includes the UAVs. Meanwhile, though the mare is not responsible for the safety of her entire herd, she is accountable for the wellbeing of her foal(s). Thus, her alertness, though not as high as that of a stallion, is increased to the point where she is still able to ensure the safety of her offsprings [27].

Therefore, height cannot be the only factor involved in our experiment. Disturbance caused by UAVs depends not only on sound intensity $(\mathrm{dB})$ and frequency $(\mathrm{Hz})$ but also by the duration and pattern of the noise. Further investigation in this area would be desirable.

As mentioned above, age plays an important role in determining the alert level of Przewalski's horses due to the nature of their role in their herds; younger horses have no need to remain vigilant, while stallions and mares have to care for their herds and foals, respectively. Furthermore, the sex of adult horses also obviously influences the distance at which they noticed foreign objects, again, due to the level of responsibility that they take on within their herds.

In terms of the practicality of unmanned aerial vehicles used as a tool in the field of animal research, our experiment leads us to believe that UAVs are an extremely useful instrument for remotely observing the behavior of certain animals. However, as has been suggested in previous experiments, UAVs must be used with caution and environmental consciousness [29]. In order to avoid disturbance to an animal's natural routine during observation, we, as scientists, must be aware and in full control of the drone's proximity to the subjects [28]. In our research, the furthest vertical distance at which the UAV was first noticed was 52 
meters. This informs us that the optimum height for observation of the Przewalski's horses will certainly be above 52 meters, though, without additional data, it will not be possible to determine the exact optimum height. Our experiment informs us that with minimized disturbance, UAVs are technologically advanced and practical scientific devices. An inconspicuously designed UAV flown at a height unnoticeable by the subject is a beneficial tool for the observation of animals in this era.

4.1. Limitations of the Study. It must be taken into account, however, that all the analyses have been performed at the individual level, and we are unable to provide a statistical analysis at the group level for this work, as it had not been anticipated before the study. However, in a group living species, the reaction of one individual may influence the behavior of the whole group. In other terms, one individual may have seen and reacted to the UAVs, ran away, and induced the same behavior in horses that did not even see the UAVs. Further research on statistical analysis at the group level might elucidate the potential reactions between individuals.

\section{Conclusion}

As a result of herd hierarchy, the various heights at which adult and immature Przewalski's horses react and flee from an unknown skyborne object is a reflection of their responses, something that may be shaped by their responsibilities and roles within their groups. Our experiment, as well as the work of countless scientists that came before us, verifies and supports this statement with data and research. We learned that the most alert horses were typically adults, while the least alert were often the immature. Furthermore, age and gender are the top two factors, first and second, respectively, that influenced the height at which each horse noticed or fled from the UAVs. In knowing the minimum distance that will cause disturbance to Przewalski's horses, we are able to better ensure that the presence of the UAV flights does not interfere with the natural behavior of these horses, thus guaranteeing a more accurate experiment. We are hopeful that, with further research and development, UAVs will become increasingly reliable and grow into a finer tool for the use of animal observation.

\section{Data Availability}

The data used and/or analysed in the study are available from the corresponding author on reasonable request.

\section{Conflicts of Interest}

The authors declare that there are no conflicts of interest.

\section{Acknowledgments}

This work was supported by Science and Technology Supporting Project of China Science Research Volunteers Alliance (CSRVA: 2019-06-ZH02). The authors would like to thank Guest Professor Zhongze Niu for supporting them to conduct the present research in the study area. The authors are also grateful to Senior Engineer Entemakh for his help on field survey. The authors are also grateful for advice given by Paul Jason Buzzard, China Exploration and Research Society, Hong Kong, China.

\section{References}

[1] C. Xia, J. Cao, H. Zhang, X. Gao, W. Yang, and D. Blank, "Reintroduction of Przewalski's horse (Equus ferus przewalskii) in Xinjiang, China: the status and experience," Biological Conservation, vol. 177, pp. 142-147, 2014.

[2] L. E. Boyd, "The behaviour of Przewalski's horses and its importance to their management," Applied Animal Behaviour Science, vol. 29, no. 1-4, pp. 301-318, 1991.

[3] W. Paige, The Remarkable Comeback of Przewalski's Horse, Smithsonial Magazine, Washington, DC, USA, 2016.

[4] I. Bouman, "The return of the Takhi," in The Tale of the Przewalski's Horse", P. Wit and I. Bouoman, Eds., pp. 82-161, KNNV Publishers, Utrecht, The Netherlands, 2006.

[5] I. Bouman and J. Bouman, "The history of Przewalski's horse," in Przewalski's Horse. The History and Biology of an Endangered Species, L. Boyd and K. Houpt, Eds., pp. 5-38, SUNY, Albany, NY, USA, 1994.

[6] S. Wakefifield, J. Knowles, W. Zimmermann, and M. C. Van Dierendonck, "Status and action plan for the Przewalski's horse (Equus ferus przewalskii)," in Equids: Zebras, Asses and Horses, P. Moehlman, Ed., pp. 82-92, IUCN Publications Services Unit, IUCN/SSC Equid Specialist Group, Cambridge, UK, 2002.

[7] P. Kaczensky, O. Ganbaatar, H. V. Wehrden, N. Enksaikhan, D. Lkhagvasuren, and C. Walzer, "Przewalski horse reintroduction in the great gobi B strictly protected area from species to ecosystem conservation," Mongolian Journal of Biological Sciences, vol. 5, no. 13-18, 2007.

[8] A. T. Bowling and O. A. Ryder, "Genetic studies of blood markers in Przewalski's horses," Journal of Heredity, vol. 78, no. 2, pp. 75-80, 1987.

[9] C. Der Sarkissian, L. Ermini, M. Schubert et al., "Evolutionary genomics and conservation of the endangered przewalski's horse," Current Biology, vol. 25, no. 19, pp. 2577-2583, 2015.

[10] S. R. B. King and J. Gurnell, "Habitat use and spatial dynamics of takhi introduced to Hustai National Park, Mongolia," Biological Conservation, vol. 124, no. 2, pp. 277-290, 2005.

[11] C. Walzer, P. Kaczensky, W. Zimmenrmann, and C. Stauffer, "Przewalski's horse reintroduction to Mongolia: status and outlook," WAZA magazine, vol. 13, no. 3-6, 2012.

[12] L. Boyd and N. Bandi, "Reintroduction of takhi, Equus ferus przewalskii, to Hustai National Park, Mongolia: time budget and synchrony of activity pre- and post-release," Applied Animal Behaviour Science, vol. 78, no. 2-4, pp. 87-102, 2002.

[13] G. Liu, A. B. A Shafer, W. Zimmermann et al., "Evaluating the reintroduction project of Przewalski's horse in China using genetic and pedigree data," Biological Conservation, vol. 171, pp. 288-298, 2014.

[14] N. Pantel, P. Leimgruber, W. Zimmermann, M. Songer, and A. Cerny, Supporting Przewalski's Horse Reintroduction Efforts in China, Development of an Integrated Conservation Project with the Wild Horse Breeding Centre in Xinjiang Province, Xinjiang, China, 2006.

[15] K. Anderson and K. J. Gaston, "Lightweight unmanned aerial vehicles will revolutionize spatial ecology," Frontiers in Ecology and the Environment, vol. 11, no. 3, pp. 138-146, 2013. 
[16] E. Marris, "Drones in science: fly, and bring me data," Nature, vol. 498, no. 7453, pp. 156-158, 2013.

[17] A. Lucieer, D. Turner, D. H. King, and S. A. Robinson, "Using an unmanned aerial vehicle (UAV) to capture micro-topography of Antarctic moss beds," International Journal of Applied Earth Observation and Geoinformation, vol. 27, pp. 53-62, 2014.

[18] C. Vermeulen, P. Lejeune, J. Lisein, P. Sawadogo, and P. Bouché, "Unmanned aerial survey of elephants," PLoS One, vol. 8, Article ID e54700, 2013.

[19] J. B. Vincent, L. K. Werden, and M. A. Ditmer, "Barriers to adding UAVs to the ecologist's toolbox," Frontiers in Ecology and the Environment, vol. 13, no. 2, pp. 74-75, 2015.

[20] L. P. Koh and S. A. Wich, "Dawn of drone ecology: low-cost autonomous aerial vehicles for conservation," Tropical Conservation Science, vol. 5, no. 2, pp. 121-132, 2012.

[21] J. A. Barasona, M. Mulero-Pázmány, P. Acevedo et al., "Unmanned aircraft systems for studying spatial abundance of ungulates: relevance to spatial epidemiology," PLoS One, vol. 9, Article ID e115608, 2014.

[22] C. Gorta'zar and J. Vicente, "Unmanned aircraft systems for studying spatial abundance of ungulates: relevance to spatial epidemiology," PLoS One, vol. 9, Article ID e115608, 2014.

[23] M. Shahbazi, J. Théau, and P. Ménard, "Recent applications of unmanned aerial imagery in natural resource management," GIScience and Remote Sensing, vol. 51, no. 4, pp. 339-365, 2014.

[24] K. Whitehead and C. H. Hugenholtz, "Remote sensing of the environment with small unmanned aircraft systems (UASs), part 1: a review of progress and challenges," Journal of Unmanned Vehicle Systems, vol. 02, no. 3, pp. 69-85, 2014.

[25] A. Hodgson, N Kelly, and D. Peel, "Unmanned aerial vehicles (UAVs) for surveying marine fauna: a dugong case study," PLos One, vol. 8, 2013.

[26] E. Vas, L. Amélie, D. Olivier, B. Guillaume, and G. David, "Approaching birds with drones: first experiments and ethical guidelines," Biology Letters, vol. 11, Article ID 20140754, 2015.

[27] M. A. Ditmer, J. B. Vincent, L. K. Werden, P. A. Iaizzo, and J. C. Tanner, "Bears show a physiological but limited behavioral response to unmanned aerial vehicles," Current Biology, vol. 25, pp. 1-6, 2015.

[28] N. M. Schroeder, P. Antonella, G. M. Romina, and C. Pablo, "An experimental approach to evaluate the potential of drones in terrestrial mammal research: a gregarious ungulate as a study model," Royal Society Open Science, vol. 7, Article ID 191482, 2020.

[29] E. A. Brunton, J. X. Leon, and S. E. Burnett, "Evaluating the efficacy and optimal deployment of thermal infrared and truecolour imaging when using drones for monitoring kangaroos," Drones, vol. 4, 2020.

[30] M. Bourjade, B. Thierry, M. Maumy, and O. Petit, "Decisionmaking in Przewalski Horses (Equus ferus przewalskii) is driven by the ecological contexts of collective movements," Ethology, vol. 115, pp. 312-330, 2009.

[31] M. Bourjade, A. de Boyer des Roches, and M. Hausberger, "Adult-young ratio, a major factor regulating social behaviour of young: a horse study," Plos One, vol. 4, 2009.

[32] J. W. Christensen, T. Zharkikh, J. Ladewig, and N. Yasinetskaya, "Social behaviour in stallion groups (Equus przewalskii and Equus caballus) kept under natural and domestic conditions," Applied Animal Behaviour Science, vol. 76, no. 1, pp. 11-20, 2002.

[33] E. S. Hogan, K. A. Houpt, and K. Sweeney, "The effect of enclosure size on social interactions and daily activity patterns of the captive asiatic wild horse (Equus Przewalskii)," Applied Animal Behaviour Science, vol. 21, pp. 147-168, 1998.

[34] L. Boyd, "The $24 \mathrm{~h}$ time budget of a takh harem stallion (Equus ferus przewalskii) pre- and post-reintroduction," Applied Animal Behaviour Science, vol. 60, no. 4, pp. 291-299, 1998.

[35] K. Krueger, G. Schneider, B. Flauger, and J. Heinze, "Contextdependent third-party intervention in agonistic encounters of male Przewalski horses," Behavioural Processes, vol. 121, pp. 54-62, 2015.

[36] S. R. B. King and J. Gurnell, "Effects of fly disturbance on the behaviour of a population of reintroduced Przewalski horses (Equus ferus przewalskii) in Mongolia," Applied Animal Behaviour Science, vol. 125, no. 1-2, pp. 22-29, 2010.

[37] L. Kolter and W. Zimmermann, "Social-behavior of Przewalski horses (Equus Przewalskii) in the Cologne zoo and its consequences for management and housing," Applied Animal Behavior Science, vol. 21, pp. 117-145, 1998.

[38] C. Feh and B. Munkhtuya, "Male infanticide and paternity analyses in a socially natural herd of Przewalski's horses: sexual selection?" Behavioural Processes, vol. 78, no. 3, pp. 335-339, 2008.

[39] N. P. Austin and L. J. Rogers, "Lateralization of agonistic and vigilance responses in Przewalski horses (Equus przewalskii)," Applied Animal Behaviour Science, vol. 151, pp. 43-50, 2014.

[40] N. P. Austin and L. J. Rogers, "Asymmetry of flight and escape turning responses in horses," Laterality: Asymmetries of Body, Brain and Cognition, vol. 12, no. 5, pp. 464-474, 2007.

[41] N. P. Austin and L. J. Rogers, "Limb preferences and lateralization of aggression, reactivity and vigilance in feral horses, Equus caballus," Animal Behaviour, vol. 83, no. 1, pp. 239-247, 2012. 